\title{
Readability, communication and terms of use of software packages
}

Gyasi, William Kodom

University of Cape Coast, Ghana (wkodomgyasi@gmail.com)

Bangmarigu, Jonah Manasseh

University of Cape Coast, Ghana (manasbangmarigu95@gmail.com)

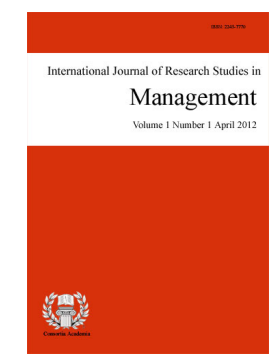

ISSN: $2243-7770$

Online ISSN: 2243-7789

Received: 29 August 2018

Revised: 28 May 2019

DOI: $10.5861 /$ ijrsm.2019.4401

Accepted: 3 June 2019

OPEN ACCESS

\section{Abstract}

Terms of Use, which is sometimes called Terms and Conditions (T\&C), are frequently unread as a result of their complexity and length. In the Information and Communication Technology (ICT) domain, it is also referred to as End User Licensing Agreement (EULA) which acts as a disclaimer between the manufacturer and the end user in the event of any malfunction. Readability formulae were used to objectively measure the comprehensibility of a document in terms of how it effectively achieves its intended purpose. End users rarely read or fully understand what they are agreeing to because of the urgency with which the software is needed. Hence, the need to evaluate the readability of terms of use of software packages to benefit both the Terms of Use of Software Packages to benefit both the end user and programmers.

Keywords: terms and conditions; End User Licensing Agreement (EULA); readability; comprehensibility; software packages 


\section{Readability, communication and terms of use of software packages}

\section{Introduction}

Communication has evolved tremendously and rapidly with the introduction of computers, and this has also impacted both written and spoken communication. The blending of computers and communication has resulted into the widely acclaimed Information and Communication Technology (ICT) era, which focuses more on technologies that deal with communication such as cell phones, the internet, wireless networks among others. The evolution of computers from First Generation Computers in the 1940's to the current Fifth Generation Computers has been characterized by various distinct features such as Vacuum Tubes for First Generation, Transistors for Second Generation, Internal Circuits (IC) for Third Generation, Very Large Scale Integration (VLSI) for Fourth Generation and smarter processing speed for Fifth Generation (Musa, 1975).

Various Operating Systems such as Microsoft, iOS, UNIX, LINUX, Android, and many others that act as an intermediary between the user and the computer hardware, use various software to command the computer (Alhazmi, Malaiyan, \& Ray, 2007). These software packages have their own advantages and disadvantages which can either help solve problems easily or adversely affect the computer system. These effects that might impede the efficacy of the computer system as a software package have made software programmers/developers/manufacturers add an agreement called Terms of Use. Thus, Terms of Use can be defined as an agreement or consensus that is reached upon before a particular product is used by the consumer. It also acts as a disclaimer on the part of the manufacturer in the event of any malfunction of a particular product. Therefore, Terms of Use is a form of communication between manufacturers of software and the potential consumers of that software. This kind of communication borders on some forms of conditions that pertain to the use of the software. Arguably, terms of use should be clear and understandable to consumers so that these consumers can make a decision as to whether they are ready to go by the terms or otherwise.

The purpose of this paper is to evaluate the readability of the Terms of Use of selected software packages. With the aid of non-proportionate stratified sampling method, a total sample of thirty eight (38) Terms of Use of different software packages for different computer operating systems was collected. The Flesch Reading Ease formula and the Flesch-Kincaid Grade Level were used to calculate the readability of the selected Terms of Use to ascertain the grade level that readers will need to effectively read and comprehend the Terms of Use of software packages. Different software from different operating systems were selected for this analysis since a user is likely to use at least one operating system. For example, a person who uses a smart phone or a computer is likely to use any of these operating systems; windows, iOS or LINUX. However, it must be noted that some software packages are not compatible with other operating systems hence the need to use different software from different operating systems.

\subsection{Objectives of the study}

$>$ To determine the readability of terms of use of the selected software packages

$>$ To determine the grade level readers require to read and understand terms of use of software packages

This study is significant in several ways. First, this study will help to establish whether there is effective communication between software manufacturers and their prospective users through terms and conditions that come with the software packages. Furthermore, the study will contribute to extant literature on readability of online text which has so far focused on various text types other than terms and conditions that accompany software packages. The study may also awaken in manufacturers of software packages to consider if there is the need for them to revise the terms and conditions that come with their software packages to ensure that end users understand what they consent to. 


\section{Literature review}

\subsection{Readability of terms of use}

Readability formulae are used widely in education and increasingly in business and government. This has generated in the generating of more than 200 readability formulae during the past thirty (30) years (Humphreys \& Humphreys, 2013). Examples of these readability formulae include Flesch Grade Level, Flesch-Kinkaid Index, Spache Readability Formula, Fog Index, New Dale-Chall Formula, SMOG Readability Formula, Coleman-Liau Index, Linsear Write Readability Formula, and many others (Greenfield, 2004; Greenfield, 1999).

According to Klare (1963, p. 3), readability is defined as "the ease of understanding or comprehending due to the style of writing". This definition focuses on separating the writing style from issues such as content, coherence and organization. On his part, McLaughlin (1969) focuses on the interaction that exists between the text and the class of readers of known characteristics such as reading skills, prior knowledge and motivation. In this paper, the Flesch Grade Level and Flesch-Kinkaid Index were used to ascertain the reading ease of software packages and the level of education needed to comprehend Terms of Use respectively.

In 1948, Rudolph Flesch propounded the Flesch Reading Ease Readability Formula in his article A New Readability Yardstick simply to assess the readability level of any written document. The Flesch Reading Ease Readability Formula focuses on the length of a sentence and the number of syllables per word. It is represented mathematically as follows:

$\mathrm{RE}=206.835-(1.015 \times \mathrm{ASL})-(84.6 \times \mathrm{ASW})$, where:

$\mathrm{RE}=$ Readability Ease (output ranges from $0-100$ );

ASL = Average Sentence Length (number of words divided by number of sentences); and

ASW = Average number Syllables per Word (number of syllables divided by the number of words)

(Dalecki, Lasora, \& Lewis, 2009).

\section{Table 1}

Range and Interpretation to calculate the readability of a document

\begin{tabular}{cc}
\hline Output Range $(\mathrm{RE})$ & Interpretation \\
\hline $90-100$ & Very Easy \\
$80-89$ & Easy \\
$70-79$ & Fairly Easy \\
$60-69$ & Standard \\
$50-59$ & Fairly Difficult \\
$30-49$ & Difficult \\
$0-29$ & Very Confusing \\
\hline
\end{tabular}

Source. Field Data, 2016.

As the name suggests, Flesch-Kincaid Index was propounded by Rudolph Flesch and John P. Kincaid. This was in 1975. It ranges from 0-100 with the higher numbers representing larger numbers of people who can comprehend the document (Humphreys \& Humphreys, 2013). Although the Flesch-Kincaid Index also uses the average number of words per sentence and average number of syllables per word, it focuses on the grade-school level or number of years of formal education a reader needs to comprehend a document. It is mathematically represented as follows:

$\mathrm{FKRA}=(0.39 \times \mathrm{ASL})+(11.8 \times \mathrm{ASW})-15.59$, where:

FKRA $=$ Flesch-Kincaid Reading Age;

ASL = Average Sentence Length (number of words divided by number of sentences); and

ASW = Average number Syllables per Word (number of syllables divided by the number of words)

(DuBay, 2004). 
Gyasi, W. K., \& Bangmarigu, J. M.

Table 2

The outputs (FKRA) can be interpreted in the table below

\begin{tabular}{cll}
\hline Score & \multicolumn{1}{c}{ School Level } & \multicolumn{1}{c}{ Interpretation } \\
\hline $100-90$ & $5^{\text {th }}$ Grade & Very easy to read. Easily understood by an average 11-year-old student \\
$90-80$ & $6^{\text {th }}$ Grade & Easy to read. Conversational English for consumers \\
$80-70$ & $7^{\text {th }}$ Grade & Fairly easy to read \\
$70-60$ & $8^{\text {th }} \& 9^{\text {th }}$ Grade & Plain English. Easily understood by 13-15 year old students \\
$60-50$ & $10^{\text {th }}$ to $12^{\text {th }}$ Grade (High School) & Fairly difficult to read \\
$50-30$ & College & Difficult to read \\
$30-0$ & College Graduate & Very difficult to read. Best understood by university graduates \\
\hline Source. Flesch, 1979. &
\end{tabular}

The results revealed that Terms of Use of software packages are so difficult to read such that on the average, one must have possessed at least a college degree in order to efficiently read and understand. In addition, it was also revealed that although readers need a substantial amount of education to comprehend the Terms of Use of software packages, readers hardly read till the last sentence. The findings suggest that Terms of Use of software packages are not communicated effectively to users therefore there is the need to make Term of Use of software packages very succinct to achieve its aim.

\subsection{Readability of web and social media content}

For as long as people have originated, shared, and studied ideas through written language, the notion of text difficulty has been an important aspect of communication and education. Hence, the need to compose text with readability in view. Readability is the ease with which a written text can be read or understood by a reader (Temnikova, Vieweg, \& Castillo, 2015).

With the advent of World Wide Web and the social networking sites, notion of determining readability of short messages is no longer limited to text messages, instructions manuals nor subtitles of movies but also social networking sites which require users to compose within certain characters limit. This limitation of number of characters for social networking sites' text has not only make conversation quick and faster, but rather, it compounded the issue of readability since users produce content with more abbreviations, acronyms and hashtags to meet characters limit policy (Davenport \& DeLine, 2014).

Social networking sites are attracting many users these days because the sites are mainly avenues for most people to gratify their information, education and entertainment needs. It is even more rewarding since users can be both users/producers of content due to the interactivity feature in these networking sites. According to Perrin (2015), average people spend 37 minutes per day on social media and 46\% of web user look towards social media when making a purchase. This is a sign that the business should use social media to drive their growth; this statement is supported by the same research that stated that out of 10 Small Medium Businesses use social media for their growth. Social media still state a high frequency of engagement. For example, about $70 \%$ of Facebook users open Facebook daily, and 45\% users open it several times a day. In Instagram have $49 \%$ of its users opening its daily including the $32 \%$ who open it several times a day (Perrin, 2015). The increase usage of social media, therefore, calls for researchers to investigate if readability of text on social media can be a barrier to text users and producers in effectively sharing information. This paper therefore reviews such papers on readability with keen interest in correlating the findings of researchers.

To begin with, the work of Temnikova et al. (2015) on The Case for Readability of Crisis Communications in Social Media focused on examining the readability of crisis communication content on the Twitter social networking site. The authors collected hundreds of tweets on crises communication posted by governments, nongovernmental organizations and main streams media from seven (7) English speaking nations. The data covered crises that occurred from 2012 to 2013: Alberta floods (Canada), Australia Bushfires (Australia), Bohol Earthquake (Philippines), Colorado floods (USA) and many others. By using Crowd Flower Experiment, the authors came out with the following findings. First, tweets written with a mixture of languages were unclear 
hence difficult to read. Also, tweets written with more acronyms have low reading ease hence are difficult to read. Likewise, tweets that mentioned the user make readability difficult. Also, the use of more hashtags especially at the beginning impact reading eases negatively. The authors recommended moderate sentence length, use of simple words and use hashtags at the end of tweets as a way to improve readability of tweets.

In terms of strengths, the authors' work was a preliminary readability study on a topic specific, thus crisis communication, in a social networking site, Twitter. The authors' findings on use of hashtags, acronyms, multilingual tweets and abbreviation as text centered variables that affect readability of tweets on twitter is quite plausible added to the knowledge on readability. Moreover, the authors used of crowd sourcing experiment as a means to collect the views of crowd workers on the difficulty of reading tweets collected on crisis communication on Twitter helped to make their research objective and inclusive of independent voices. Another strength of the work is that the authors employed mixed method approach. With the qualitative, the researchers through content analysis of the sampled tweets discovered the tweets were difficult to read. However, due to the tweets text centered variables that are not measurable by traditional readability formula, the authors adopted the Crowd Flower Experiment to collect the views of participants in other to validate the results. The participants' views confirmed the result that the sampled tweets were difficult to read and they went ahead to provide suggestions such as removal of hashtags, initials and other text centered variables as the possible way to make tweets readable.

Notwithstanding, the authors' work is limited in terms of scope since it covered only one social networking site, Twitter, and few English speaking countries. This might be due to the fact that the authors' work is preliminary study focusing on the possibility of readability failure in communicating crisis on social networking sites. Perhaps, subsequent research can dive into researching more than one social networking site especially Facebook and WhatApp which allow more characters than twitter. Moreover, the authors could have used, at least if not the traditional metrics, the computational metrics such as GUI evaluator or Age Rank algorithm to measure their sampled tweets to know the score instead of only content analysis.

Another work by Davenport and DeLine on the Readability of Tweets and Their Geographic Correlations with Education also exposed some interesting textual features of tweets that can affect tweets readability on the social networking site. The co-authors obtained 49 million tweets directly from twitter and from which they selected 17.4 million tweets, written in the English language, for their study. The co-authors used a Flesch Reading Ease formula to determine a corpus of 17.4 million tweets. Comparing the results of the tweets and other short messages such as text messages and chats, the authors discovered that tweets have difficult readability scores compared to other short format communication, such as SMS or chat. This linguistic difference is insensitive to the presence of "hashtags" within tweets. Their findings also revealed that including hashtags in the reading ease calculation overall makes tweets harder to read, exactly in line with Temnikova's, et al. (2015) findings.

Moreover, by utilizing geographic data provided by $2 \%$ of users, joined with "Zip Code Tabulation Are (ZCTA)" level of education data from the U.S. Census, they found an intriguing correlation between the average readability and the college graduation rate within a ZCTA. These points towards a difference in either the underlying language, or a change in the type of content being tweeted in these areas.

Davenport and DeLine work is rich in content in that their work further probes how abbreviations and site specific style of writing has impacted the readability of twitter messages negatively. Furthermore, the co-authors used Flesch reading formula in their analysis. This formula has been reckoned to be reliable and widely used in readability studies. The authors' also finding correlation in terms of education, geographical location and readability of tweet produced is quite remarkable.

Notwithstanding, the co-authors use of Flesch reading ease, which is although improved, it still lacks the metrics to measure site related textual features such as abbreviations, acronyms and hashtags. It is therefore maybe perhaps the reason why the authors included threats to validity section in their submission to talk about 
site centered variables such as abbreviations and acronyms and special words such as lololol, hahaha, and many others which cannot be measured by the Flesch reading scale hence affecting the readability score the authors obtained in their study. In such a case, the co-authors could have adopted a computational readability formula to back the lapses of Flesch reading scale or better still elicit the views of participants whom they will give the sample text.

Also, Readability Assessment of Policies and Procedures of Social Networking Sites authored by Meiselwitz (2013) is a work that examines the readability of social networking sites policies and procedures. According to Meiselwitz (2013) many internet users are today members of social network sites, building personal profiles and interacting with millions of users worldwide. These virtual environments are based on Web 2.0 technology and offer rich user interaction, personalized use of the environment, and option for sophisticated user-created content.

Social media environments have developed into large communities with complex relationships within the community, which are covered by the policies and procedures (Meiselwitz, 2013). The author examined the accessibility and readability of social networking sites policies and procedures. The author discovered that policies and procedures of social media sites are normally difficult for readers at various level because of the length, file format and placement of the policies and procedures in the site. The study also unearth that, readers mostly scan through policies and procedures or read arts and ignore the rest because of the text difficulty to read. Also, the author discovered that changes to policies and procedures, especially default setting changes, are not normally welcome and noticed by users because policies are not accessible at the user interface and involves much time to read and understand. In cases where users read, they find it difficult to apply the policies and procedures due to text difficulty which sometimes leads to sites manages imposing the changes on users.

The work is plausible in that it examines a part of readability of microblogging services that cut across most social media sites. And since, sites policies and procedures bind users and developers of sites together, the need to make policies and procedures accessible and readable is key for readability studies. The author use of 24 social networking sites is very inclusive as compare to other readability on social network sites that focuses on one site.

From these studies, one can infer that twitter as social networking site readability can be marred if users use hashtags indiscriminately, use acronyms excessively and use abbreviations known personally. Moreover, the use of mix language in tweets can affect readability. Even though Meiselwitz (2013) examined the accessibility and readability of social networking sites policies and procedures, the work did not explore the terms of use of these social networking software packages. Since every software come with the terms of use, it is vital to investigate how readable the terms of use of software packages for the prospective users.

\subsection{Terms of Use}

A Term of Use or End User Licensing Agreement (EULA) of a software package is an important legal agreement between the programmer and the user which the user must agree with and accept before installing or using the software. Terms of Use are structured based on the purpose and other legal agreement of the software company; however, certain common features are mostly found in a standard term of use. These are

$>$ Introduction

$>$ Licensing of Use

$>$ Restrictions of Use

$>$ Termination of Use

$>$ Limitation of Liability

$>$ Disclaimers of Warranties

$>$ Copyright Infringement

$>$ Contact Information

Every Term of Use begins with an introduction, which gives basic details such as the name of the software, 
Readability, communication and terms of use of software packages

the name of the manufacturer and any other names or affiliates that might fall under the scope of the terms.

Licensing of Use - explicitly grants users the authorization to use the software and the scope of the granted license.

Restrictions of Use - also explains vividly the extent to which a user is restricted in using the software package. This includes copying any portion of the software as your own, using the software in violation of its application laws, and many others.

Termination of Use- states that "if a user violates the Term of Use or any other agreements the developer(s) might have and wish to include, the license will be terminated and the software removed from the user's device".

Limitation of Liability - basically lets the users know that the programmer/developer/manufacturer is not to be held responsible for any damages that might arise out of using the software on the user's device.

Disclaimers of Warranties - make it clear to the user that the manufacturer will not warrant anything beyond the minimum required by law or stated in the Term of Use.

Copyright Infringement - informs the user that all the materials used in the preparation of the software are protected under copyright laws and that no ownership right or right to use any trademarks is granted to the user with their license.

Contact Information - provides the user with the contact details of the manufacturer such as website, mailing address, telephone numbers, email address, and many others.

\subsection{Theoretical framework}

In the words of Pikulski and Chard (2005), 'As part of a developmental process of building decoding skills, fluency can form a bridge to reading comprehension'. As the co-authors cited the definition of Reading Panel (National Institute of Child Health and Human Development [NICHD], 2000), fluency is "the ability to read text quickly, accurately, and with proper expression" (NICHD, 2000, p. 3-5). However, the authors pointed out that such a definition emphasizes fluency as an oral reading phenomenon and this has limited the attention given to fluency in reading comprehension. According to Harris and Hodges, (1985, p85), fluency is "freedom from word identification problems that might hinder comprehension. This definition of fluency has enlarged it to cover comprehension. It is therefore on this notion of fluency that pioneers of fluency theory such as Samuel (2002), Stecker, Roser, and Martinez (1998), based their postulations.

According to Stecker, Roser, and Martinez (1998, p. 306).), "Fluency has been shown to have a 'reciprocal relationship' with comprehension, with each fostering the other". Therefore reading fluency refers to efficient, effective word recognition skills that permit a reader to construct the meaning of a text. Pikulsi and Chard (2005) identified two construct of fluency which are surface construct and deep construct. A surface construct of fluency builds on an oral prosody of oral reading while a deep construct views fluency far more broadly as part of a developmental process of building decoding skills that will form a bridge to reading comprehension and that will have a reciprocal, causal relationship with reading comprehension. Fluency builds on a foundation of oral language skills, phonemic awareness, familiarity with letter forms, and efficient decoding skills.

Ehri's theory of stages of reading development and fluency is one elegant theory on fluency. According Ehri (1995), there are four stages of reading development and fluency which are Pre-alphabetic stage, Partial alphabetic stage, Fully alphabetic stage and Skilled reading level. At the pre-alphabetic stage, readers lack understanding of alphabetic principle which is letters and their sounds and hence have difficulty pronouncing and except by doing association of letters based on their visual componets such as Monkey, the ' $y$ ' tail represents the monkey's tail. This is problematic if there is error in the visual association such as 'my'. In the partial alphabetic stage, readers learn the letters and their sounds but their knowledge of sounds are limited hence they 
can find it difficult to pronounce unfamiliar words. Fully alphabetic stage is where readers have the ability to use pronunciation and hence can pronounce unfamiliar words based on the sounds combinations. This however, may not be fluent readers as in reading fast. The skilled level is where readers develop the skill of knowing words by sight. At this stage, readers can read fast. Ehri (1998) identified building graph phonic foundations for fluency. These are letter familiarity; phonemic awareness and knowledge of graphemes typically represent phonemes in words. Ehri's theory made the decoding process as dependent on readers' ability to develop their reading fluency.

One aspect of Ehri's postulation that is of great importance to readability studies is the addition of language skills to graph phonic skills as a requirement for success in fluency of reading comprehension among readers. Ehri's (1998) 'theory requires a foundation in language skills so that students (readers) are familiar with the syntax or grammatical function of the words and phrases they are reading and with their meanings. According to Ehri (1998), one of the greatest challenges facing educators is developing the oral language and vocabulary skills of children, particularly those who are learning English as a second language or those who spent their preschool years in language-restricted environment. She further asserts that highly frequently used words such as the, of, at among others help readers develop vocabulary skills.

The relevance of fluency theory to the present study is therefore clear. A readable text is a text that is composed with readers in mind. The author of the text tries to make use of familiar words and phrases as well as plain language, so as to enhance readers' comprehension. Ehri's (1995) fluency theory is therefore apt as the theoretical framework for this study.

\section{Methodology}

\subsection{Research design, sample and sampling techniques}

The researcher used the quantitative research approach in collecting and analysing data. The researcher opted for quantitative research design because the design concerns the relationships within measurable variables with the intent to explain, predict and control a phenomenon (Leedy, 1993). A further reason for using quantitative research approach is that in the field of ICT, this approach often deals with results computation and system analysis using scientific approach (Kumar, 2005).

A total of thirty eight (38) Terms of Use of software packages constituted the sample for the study. These Terms of Use of software packages were compiled from the following Operating Systems: Windows, iOS and LINUX. The non-proportionate stratified sampling method employed for the study. This was as a result of the heterogeneous nature of the software packages of the various Operating Systems under study. Some of the software packages are compatible with others. This ensured a high degree of representativeness of all the strata in the targeted population, though it was time consuming and tedious. However, it also reduced bias that could have been introduced into the sample as a result of non-equal numbers of the selected Terms of Use of software packages.

\subsection{Data collection}

Thirty eight (38) Terms of Use of software packages were first screenshot in the process of its installation. These screenshots were later converted into pdf files using http://www.convertpdfsnow.comand in order to extract the actual text needed for the readability index analysis to calculate readability score using Microsoft Word. According to Shehadeh and Strother (1994), the preparation stage for computerized calculation of readability of a document requires an editing of the entire document to be in conformity with the original document. The editing stage involves the proper usage of punctuation marks such as commas and the removal or exclusion of full stops in abbreviated forms of words, which is interpreted by the computer as the end of a sentence (DuBay, 2007). In the calculations for the readability of the Terms of Use of the software packages, phrases and subordinate clauses in the form of titles, headings and bulleted points that were not expressing a 
complete thought were exempted from the analysis. In order to attain accurate readability scores, careful scrutiny was done during the preparation stage for readability calculation (Armbruster, Osbon, \& Davisonet, 1985).

\section{Discussions and analysis of data}

Table 3

List of selected software packages and its compatible operating systems

\begin{tabular}{|c|c|c|c|}
\hline Software Packages & Windows & $\mathrm{iOS}$ & LINUX \\
\hline Acrobat X & $\checkmark$ & $\checkmark$ & \\
\hline Adobe Media Player & $\checkmark$ & $\checkmark$ & \\
\hline ArcSoft Webcam Companion & $\checkmark$ & $\checkmark$ & \\
\hline Ashampoo & $\checkmark$ & $\checkmark$ & $\checkmark$ \\
\hline Cyber Link DVD & $\checkmark$ & & \\
\hline Daemon Tools Lite & $\checkmark$ & & \\
\hline Duplicate Files Fixer & $\checkmark$ & $\checkmark$ & $\checkmark$ \\
\hline Facetime & & $\checkmark$ & \\
\hline Firefox & $\checkmark$ & $\checkmark$ & \\
\hline GOM Player & $\checkmark$ & $\checkmark$ & \\
\hline ICQ & $\checkmark$ & $\checkmark$ & \\
\hline iWork & & $\checkmark$ & \\
\hline Jet Audio & $\checkmark$ & $\checkmark$ & $\checkmark$ \\
\hline KODi & $\checkmark$ & $\checkmark$ & \\
\hline Logic Pro & & $\checkmark$ & \\
\hline Mavis Beacon & $\checkmark$ & & \\
\hline Mine Plex & $\checkmark$ & $\checkmark$ & \\
\hline Music Match & $\checkmark$ & $\checkmark$ & $\checkmark$ \\
\hline Nero & $\checkmark$ & $\checkmark$ & $\checkmark$ \\
\hline Partition Magic & $\checkmark$ & & $\checkmark$ \\
\hline PCDJ & $\checkmark$ & & $\checkmark$ \\
\hline Podcast & & $\checkmark$ & \\
\hline Quick Time & $\checkmark$ & $\checkmark$ & \\
\hline Real Player & $\checkmark$ & $\checkmark$ & $\checkmark$ \\
\hline Skype & $\checkmark$ & $\checkmark$ & $\checkmark$ \\
\hline SpaceSniffer & $\checkmark$ & $\checkmark$ & \\
\hline Spybot & $\checkmark$ & $\checkmark$ & $\checkmark$ \\
\hline Tomb Raider & $\checkmark$ & $\checkmark$ & $\checkmark$ \\
\hline Torment & $\checkmark$ & $\checkmark$ & $\checkmark$ \\
\hline Utorrent & $\checkmark$ & $\checkmark$ & $\checkmark$ \\
\hline Virtual DJ & $\checkmark$ & $\checkmark$ & \\
\hline VLC & $\checkmark$ & $\checkmark$ & $\checkmark$ \\
\hline VOX Player & $\checkmark$ & $\checkmark$ & $\checkmark$ \\
\hline Windows 10 & $\checkmark$ & & \\
\hline Windows Movie Maker & $\checkmark$ & & \\
\hline WinRAR & $\checkmark$ & $\checkmark$ & \\
\hline Wunderlist & & $\checkmark$ & \\
\hline Zoom Player & $\checkmark$ & $\checkmark$ & $\checkmark$ \\
\hline
\end{tabular}

Source. Field Data, 2016.

Table 3 presents the list of software packages that were selected as the sample for the analysis. The researcher also grouped these software packages based on the operating systems that are compatible with the software. The software packages come in the form of office applications, music players, computer utilities, file extractors, and many others.

The three operating systems were chosen because they are the most widely used operating systems for computers. 
Gyasi, W. K., \& Bangmarigu, J. M.

\section{Table 4}

Scores of selected software packages using Flesch Reading Ease and Flesch-Kincaid Grade Level

\begin{tabular}{|c|c|c|}
\hline Software Packages & Flesch Reading Ease & Flesch-Kincaid Grade Level \\
\hline Acrobat X & 26.9 & 15.7 \\
\hline Adobe Media Player & 27.6 & 16.1 \\
\hline ArcSoft Webcam Companion & 27.6 & 14.7 \\
\hline Ashampoo & 63.0 & 7.7 \\
\hline Cyber Link DVD & 36.1 & 14.9 \\
\hline Daemon Tools Lite & 30.4 & 15.6 \\
\hline Duplicate Files Fixer & 29.6 & 14.8 \\
\hline Facetime & 54.1 & 7.4 \\
\hline Firefox & 40.2 & 12.9 \\
\hline GOM Player & 33.5 & 15.3 \\
\hline ICQ & 30.2 & 16.4 \\
\hline iWork & 33.3 & 13.7 \\
\hline Jet Audio & 43.7 & 8.7 \\
\hline KODi & 61.4 & 8.7 \\
\hline Logic Pro & 50.6 & 7.6 \\
\hline Mavis Beacon & 29.9 & 15.3 \\
\hline Mine Plex & 60.6 & 8.6 \\
\hline Music Match & 34.5 & 15.4 \\
\hline Nero & 31.8 & 14.3 \\
\hline Partition Magic & 42.7 & 11.2 \\
\hline PCDJ & 37.0 & 13.8 \\
\hline Podcast & 43.7 & 11.3 \\
\hline Quick Time & 26.0 & 16.6 \\
\hline Real Player & 30.4 & 14.5 \\
\hline Skype & 54.8 & 8.5 \\
\hline SpaceSniffer & 47.1 & 14.7 \\
\hline Spybot & 30.3 & 15.3 \\
\hline Tomb Raider & 19.2 & 20.2 \\
\hline Torment & 41.5 & 12.8 \\
\hline Utorrent & 22.7 & 17.6 \\
\hline Virtual DJ & 36.8 & 13.6 \\
\hline VLC & 44.4 & 11.2 \\
\hline VOX Player & 33.0 & 15.5 \\
\hline Windows 10 & 42.2 & 12.2 \\
\hline Windows Movie Maker & 18.2 & 17.7 \\
\hline WinRAR & 42.2 & 12.2 \\
\hline Wunderlist & 38.5 & 13.0 \\
\hline Zoom Player & 32.5 & 15.5 \\
\hline
\end{tabular}

Source. Field Data, 2018.

\section{Data analysis and discussion}

The decision by the researcher to shortlist and select only thirty eight (38) software packages was based on a survey conducted among students of the University Cape Coast, students of the University Practice Senior High School, and members of the general public who have personal computers. After the survey, it was revealed that out of the 200 people interviewed, 111 people, representing 55.5\% use Microsoft Operating System. 59 people, representing $29.5 \%$, use iOS, and LINUX contributed $15 \%$, which makes up 30 people.

Software packages that were compatible with all the three operating systems were considered first for the analysis, followed by those compatible with two operating systems and lastly, one software package designed for a particular operating system. It was revealed that most of the Microsoft and LINUX software packages were compatible with all the three operating systems; however, most of the software packages of iOS are designed purposely for Apple products and as such compatible with iPad, iPhone and iMac. 


\section{Table 5}

Flesch Reading Ease scores of the selected software packages

\begin{tabular}{|c|c|c|}
\hline Software Packages & Flesch Reading Ease & Interpretation \\
\hline Acrobat X & 26.9 & Very confusing \\
\hline Adobe Media Player & 27.6 & Very confusing \\
\hline ArcSoft Webcam Companion & 27.6 & Very confusing \\
\hline Ashampoo & 63.0 & Standard \\
\hline Cyber Link DVD & 36.1 & Difficult \\
\hline Daemon Tools Lite & 30.4 & Difficult \\
\hline Duplicate Files Fixer & 29.6 & Difficult \\
\hline Facetime & 54.1 & Fairly difficult \\
\hline Firefox & 40.2 & Difficult \\
\hline GOM Player & 33.5 & Difficult \\
\hline ICQ & 30.2 & Difficult \\
\hline iWork & 33.3 & Difficult \\
\hline Jet Audio & 43.7 & Difficult \\
\hline KODi & 61.4 & Standard \\
\hline Logic Pro & 50.6 & Fairly difficult \\
\hline Mavis Beacon & 29.9 & Very confusing \\
\hline Mine Plex & 60.6 & Standard \\
\hline Music Match & 34.5 & Difficult \\
\hline Nero & 31.8 & Difficult \\
\hline Partition Magic & 42.7 & Difficult \\
\hline PCDJ & 37.0 & Difficult \\
\hline Podcast & 43.7 & Difficult \\
\hline Quick Time & 26.0 & Very confusing \\
\hline Real Player & 30.4 & Difficult \\
\hline Skype & 54.8 & Fairly difficult \\
\hline SpaceSniffer & 47.1 & Difficult \\
\hline Spybot & 30.3 & Difficult \\
\hline Tomb Raider & 19.2 & Very confusing \\
\hline Torment & 41.5 & Difficult \\
\hline Utorrent & 22.7 & Very confusing \\
\hline Virtual DJ & 36.8 & Difficult \\
\hline VLC & 44.4 & Difficult \\
\hline VOX Player & 33.0 & Difficult \\
\hline Windows 10 & 42.2 & Difficult \\
\hline Windows Movie Maker & 18.2 & Very confusing \\
\hline WinRAR & 42.2 & Difficult \\
\hline Wunderlist & 38.5 & Difficult \\
\hline Zoom Player & 32.5 & Difficult \\
\hline
\end{tabular}

Source. Field Data, 2018.

\subsection{Objective 1: To determine the readability of terms of use of selected software packages}

The table above shows the Flesch reading ease scores of each of the terms of use of the selected software packages. Flesch reading ease is a time tested and reliable readability metric that gives reliable and accurate measure of readability of a text. It is succint from the table that 8 of the terms of use of the software packages were very confusion to read and these are Acrobat X, Adobe Media player, Arcsoft Webcam Companion, Mavis Beacon, Quick Time, Tomb Raider, Utorrent and Window Movie Maker. These terms of use of software packages readability scores are within the range of 0-29 and according to the Flesch reading scale, this range is very difficult to read or very confusion. 24 of the terms of use of the software packages were difficult to read. These software packages had scores within the range of 30-49. 3 of the terms of the use of the software packages were fairly difficult. These three packages had scores within 50-59. These three fairly difficult to read packages were 
Gyasi, W. K., \& Bangmarigu, J. M.

Skype, Logic Pro, and Face time. 4 of the terms of use of the software packages had standard readability score. These softwares packages are Ashampoo, KODi and MinePlex. It is obvious that majority of the terms of use of software packages are difficult to read to the average who is a potential user of these software packages.

This result is consistent with Temnikova et al. (2015) and Davenport and DeLine (2014) findings' that tweets, chats and other short messages are difficult to read. Also, Meislewitz (2013) discovered that policies and procedures of social media sites are normally difficult for readers at various levels because of the length, file format and placement of the policies and procedures in the site. These scores imply that out of the thirty eight sampled packages only three packages' terms of use are written for public readership. This is alarming since software packages users are diverse groups with difference abilities in terms of reading. It will be preferable and safer for manufacturers to compose terms of use at the standard or fairly easy to read level since majority of software users depend on these terms of use to make informed decisions about whether to use these software packages or not.

\subsection{Objective 2: To determine the grade level readers require reading and understanding terms of use of software packages.}

While the Flesch reading ease provides the readability scores of the packages in a range of very easy to very difficult read scale (100-0 respectively), the Flesch-Kincaid Grade level determines the grade level readers need to read and understand the terms of use of the software packages. In other words, the Flesch Kincaid Grade level translates the Flesch reading ease scores to the number of years of education readers have to acquire in order to read and understand the terms of use. This measure is important to predict what particular age group or educational level will find the terms of use suitable to read and understand. From table 6, the figures show that 7 of the software packages' terms of use require readers to have at least 16 years of education which is equivalent to graduate level of education. It means that a reader should at least be a university graduate in order to find text familiar and easy to read. 17 terms of use of the selected software packages require a reader to have at least 13 years to 15 years of education in order to find terms of use readable and understandable. For these of group of packages, a college student can read and understand the content. This means that readers below college level will find this category of terms of use of software packages difficult to read and understand. 6 of the terms of use of the software packages required 10 to 12 years of education for a reader to read and understand. In other words, a high school student may read and understand such terms of use according to the Flesch-Kincaid Grade Level score. 7 software packages required 7 to 9 years of education in order for readers to read and understand. This implies that this category of terms of use of software packages is readable to reeaders with $7^{\text {th }}$ to $9^{\text {th }}$ grade level. 8th grade is the recommended reading grade level for public documents. However, only four of the selected softwares packages, which are Skype, Mine Plex, Jet Audio, and Kodi, were written at the recommended $8^{\text {th }}$ grade level for public documents. It means that these terms of use are readable to majority of the public, if not all.

In contrast, Tomb Raider scored 20 which mean that a reader requires 20 years of education in order to read and understand. This score matches with post graduate level of education and therefore is very difficult to read for the majority of users. Since terms of use of software packages are targeting readers of varied educational levels, it is expedient to stick to the $8^{\text {th }}$ grade level which is appropriate for public documents. From the results, it obvious that readers below $12^{\text {th }}$ grade will find terms of use of the selected software packages difficult to read. This is similar to Meislewitt's (2013) finding that readers require $12^{\text {th }}$ grade and above to read and understand policies and procedures of social media sites. This trend is worrying given the importance of these texts not only to users of software but also manufacturers of the software packages.

As posited by Ehri in her Fluency theory, readers of the pre-alphabetic stage, partial alphabetic stage and fully alphabetic stage will find the terms of use difficult to read since most of the words may not be familiar to readers at these levels. Therefore, to make the terms of use readable, terms of use's text should be composed with readers in mind. This requires the terms of use authors to use of familiar words and phrases as well as plain 
language, so as to enhance readers' comprehension as recommended by Ehri in her fluency theory. Terms of use authors should not be oblivion of the fact all readers are not at the skilled level where most words are familiar by sight hence text of terms of use should consider the reading categories or stages in order to prepare readable terms of use that serve their purpose and meet audience' information needs.

\section{Conclusions}

In this study, the researcher examined the readability of terms of use of software packages. First, the study explored the level of readability of selected terms of use of software packages. From the results of Flesch reading ease scores, the study showed that 30 out of the sampled 38 terms of use of software packages are difficult to read. Also, using the Flesch-Kincaid reading ease, the researcher further discovered that readers need to attain at least $12^{\text {th }}$ grade in order to read and understand the terms of use of the software packages.

In a nutshell, Terms of Use of software packages are difficult to read, and understanding the nature of Terms of Use depends on the number of users. When the number of users of a particular software package is large, there is the tendency to involve more features in the terms of use in order to avoid legalities in case of malfunction. This might also be the reason why users do not actually spend time to read the terms of use till the end since the software package might have been recommended by an already user. Again, it might also be that they are in a hurry to use the software package.

This study serves as a major attempt at exploring and examining effective communication in the Information and Communication Technology (ICT). This study will sensitize and encourage software programmers to be succinct, brief and concise in their choice and use of words and sentences in a way that will make terms of use of software packages user friendly. This involves using plain language and avoiding excessive use of unfamiliar words. This will make users understand Terms of Use of software packages which will eventually attract larger reader-base. Finally, this paper will provide the basic foundation for software programmers to devise new ways of writing terms of use for software packages that are guided by linguistic considerations to ensure effective communication for readers understanding.

\section{References}

Alhazmi, O. H., Malaiya, Y. K., \& Ray, I. (2007). Measuring, analyzing and predicting security vulnerabilities of softwares. Computers \& Security, 26(3), 219-228. https://doi.org/10.1016/j.cose.2006.10.002

Armbruster, B. B., Osborn, J. H., \& Davison, A. I. (1985). Readability formulas may be dangerous to your textbooks. Educational Leadership, 1, 18-20.

Creswell, J. W. (2003). Research design: Qualitative, quantitative and mixed methods approaches (2nd ed.). New York: Thousand Oaks, CA Sage.

Dalecki, L., Lasora, D. L., \& Lewis, S. C. (2009). The new readability problem. Journalism Practice, 3(1), 1-12. https://doi.org/10.1080/17512780802560708

Davenport, J. R., \& DeLine, R. (2014). The readability of tweets and their geographic correlation with education. Retrieved from http://wing.nus.edu.sg:8080/SMCorpus/

DuBay, W. H. (2004). The principles of readability. Costa Messa, California: Impact Information.

DuBay, W. H., (2007). Smart language: Readers readability and the grading of text. Costa Messa, California: Impact Information.

Ehri, L. C. (1995). Stages of development in learning to read words by sight. Journal of Research in Reading, 18, 116-125. https://doi.org/10.1111/j.1467-9817.1995.tb00077.x

Ehri, L. C. (1998). Grapheme-phoneme knowledge is essential for learning to read words in English. In J. L. Metsala \& L. C. Ehri (Eds.), Word recognition in beginning literacy (pp. 3-40). Mahwah, NJ: Erlbaum.

Eltorai, A. E., Naqvi, S. S., Ghanian, S., Eberson, C. P., Weiss, A. P., Born, C. T., \& Daniels, A. H. (2015). Readability of invasive procedure consent forms. Clinical and Translational Science, 8(6), 830-833. https://doi.org/10.1111/cts.12364 
Gyasi, W. K., \& Bangmarigu, J. M.

Flesch, R. (1979). How to write plain English. Boston: Harper Collins Publishers.

Greenfield, G. R. (1999). Classic readability formulas in a EFL context: Are they valid Japanese Speakers? (Unpublished doctoral dissertation). Temple University, Philadelphia, PA United State.

Greenfield, J. (2004). Readability formulas for EFL. JALT Journal, 26, 5-24.

Gyasi, W. K., (2013). The role of readability in science education in Ghana: A readability index analysis of Ghana association of science teachers textbooks for senior high school. IOSR Journal of Research \& Method in Education, 2(1), 9-19. https://doi.org/10.9790/7388-0210919

Harris, T. L., \& Hodges, R. E. (1995). The literacy dictionary: The vocabulary of reading and writing. Newark, DE: International Reading Association.

Humphreys, A. H., \& Humphreys, J. L. (2013). Reading difficulty levels of selected articles in the journal of research in music education. Music Education Research International, 2, 15-25.

Hussain, W., Sohaib, O., Ahmed, A., \& Khan, Q. M. (2011). Web readability factors affecting users of all ages. Australian Journal of Basic and Applied Sciences, 5(11), 972-977.

Klare, G. (1963). The measurement of readabiliy. Ames, Iowa: Iowa State University Press.

Kumar, R. (2005). Research methodology: A step-by-step guide for beginners ( $2^{\text {nd }}$ ed.). London: SAGE Publications.

Leedy, P. D. (1993). Practical research: planning and design. New Jersey: Prentice-Hall.

Lively, B. A., \& Pressey, S. L. (1923). A method for measuring the vocabulary burden of textbooks. Educational Administration and Supervision, 73(9), 389-398.

Manning, D. (1981). Writing readable health messages. Public Health Reports, 96(5), 464-465.

McLaughlin, G. H. (1969). SMOG grading: A new readability formula. Journal of Reading, 22, 639-646.

McLaughlin, G. H. (1974). Temptations of the Flesch. Instructional Science, 2(4), 367-384. https://doi.org/10.1007/BF00123459

Meiselwitz, G. (2013). Readability assessment of policies and procedures of social networking sites. In A. Ozok \& P. Zaphiris (Eds.), Online communities and social computing (pp. 67-75). Berlin: Springer. https://doi.org/10.1007/978-3-642-39371-6_8

Musa, J. D. (1975). A theory of software reliability and its application, IEEE Transactions on Software Engineering, 1(3), 312-327. https://doi.org/10.1109/TSE.1975.6312856

Perrin, A. (2015). Social media usage: 2005-2015. US, Washington: Pew Research Center. Retrieved from https://www.pewinternet.org/2015/10/08/social-networking-usage-2005-2015/

Pikulski, J., \& Chard, D. J. (2005). Fluency: Bridge between decoding and reading comprehension. The Reading Teacher, 58(6), 510-519. https://doi.org/10.1598/RT.58.6.2

Samuels, S. J. (1979). The method of repeated readings. The Reading Teacher, 32, 403-408.I

Samuels, S. J. (2002). Reading fluency: its development and assessment. In A. E. Farstrup \& S. J. Samuels (Eds.), What research has to say about reading instruction (3rd ed., pp. 166-184). Newark, DE: International Reading Association. https://doi.org/10.1598/0872071774.8

Shehadeh, C. M., \& Strother, J. B. (1994). The use of computerized readability formulas: Bane or blessing? Annual Conference-Society for Technical Communication, 41, 225-227.

Temnikova, I., Vieweg, S., \& Castillo, C. (2015). The case for readability of crises communication in social media. International World Wide Web Conference Committee. https://dx.doi.org/10.1145/2740908.2741718 